\title{
Efficient and accurate electromagnetic scattering analysis of perfectly conducting thick plates
}

\author{
Eduard Ubeda*, Ivan Sekulic and Juan M. Rius \\ CommSensLab, Signal Theory and Commnunications Department (TSC) \\ UniversitatPolitècnica de Catalunya (UPC) \\ Barcelona, Spain \\ ubeda@tsc.upc.edu
}

\begin{abstract}
The scattering analysis with the Electric-Field Integral Equation by the Method of Moments of perfectly conducting plates is normally carried out through the thin-plate approximation, which models the closed surface as an open surface and discards the influence of the plate rim. The resulting accuracy is accepted in many practical applications as long as the thickness of the plate is small enough. However, in the scattering analysis of plates with grazing incidences, the thin-plate approximation gives rise to severe inaccuracies. In this paper, we present a new numerical approach, which we name thick-plate formulation, for the accurate computation of the current and the RCS for a plate with nonzero thickness. Our scheme provides similar accuracy as the solution obtained with the full modelling of the plate and with less computational time.
\end{abstract}

\section{INTRODUCTION}

The electromagnetic scattering analysis of perfectly conducting (PEC) plates with very small thickness is normally undertaken through the thin-plate approximation [1], which stems from the application of the surface equivalence principle as the plate thickness tends to zero. In the limit, the ElectricField and the Magnetic-Field equations, EFIE and MFIE, degenerate into problems where the unknowns involve, respectively, the sum or the subtraction of the currents over the top and bottom faces of the plate [2]. The plate is then modelled as an open surface and the scattering effect of the rims is neglected. In practice, the thin-plate approximated EFIE is adopted for such cases because the scattered fields depend on the addition of the top and bottom currents of the plate. The thin-plate approximation becomes very advantageous because of the drastic reduction of unknowns with respect to the full modelling of the plate as a closed surface. Although some accuracy is sacrificed, the thin-plate approximation has been traditionally implemented in many practical applications. However, in cases where the effect of the plate rim on the scattering pattern becomes critical, such as the scattering analysis of plates with grazing incidences, the thin-plate approximation becomes inaccurate. In this paper, we present a new efficient formulation for the accurate scattering analysis of plates with nonzero thickness under an arbitrary incidence.

\section{THICK-PLATE FORMULATION}

Consider a free-standing square plate with side length $L$ and nonzero thickness $\delta$ under an impinging plane wave. The analysis by the method of moments (MoM) and the EFIE requires the rectangular meshing of the surface $S$ around the plate and the expansion of the current with the rooftop basis functions, $\left\{\boldsymbol{t}_{n}\right\}$, [3]. The approximated scattered electric field $\tilde{\boldsymbol{E}}^{s}$ then becomes

$$
\begin{aligned}
\tilde{\boldsymbol{E}}^{s}(\boldsymbol{r})= & -j k \eta \sum_{n=1}^{N_{E}} c_{n} \iint_{Q_{n}} G\left(\boldsymbol{r}, \boldsymbol{r}^{\prime}\right) \boldsymbol{t}_{n}\left(\boldsymbol{r}^{\prime}\right) d S^{\prime} \\
& -j \frac{\eta}{k} \sum_{n=1}^{N_{E}} c_{n} \iint_{Q_{n}} \nabla G\left(\boldsymbol{r}, \boldsymbol{r}^{\prime}\right) \nabla^{\prime} \cdot \boldsymbol{t}_{n}\left(\boldsymbol{r}^{\prime}\right) d S^{\prime}
\end{aligned}
$$

where $Q_{n}$ represents the duplet of rectangular facets sharing the $n$-th edge and $\left\{c_{n}\right\}$ stands for the set of $N_{E}$ unknowns $\left(N_{E}\right.$ stands for the number of mesh edges). The parameters $k$ and $\eta$ denote the wavenumber and the free-space impedance, whereas $G$ represents the free-space Green's function. The resulting matrix system arises from the rooftop testing over the rectangular tessellation over $S$ of the following electric-field condition

$$
\left.\tilde{\boldsymbol{E}}^{s}(\boldsymbol{r})\right|_{\tan , \boldsymbol{r} \in S}=-\left.\boldsymbol{E}^{i n c}(\boldsymbol{r})\right|_{\tan , \boldsymbol{r} \in S}
$$

where $\boldsymbol{E}^{\text {inc }}$ denotes the incident electric field.

Our thick-plate formulation applies the tangential-electric condition in (2) only over the $N_{R}$ pairs of rectangles sharing mesh edges that lie inside the plate rim or that match the outer boundary line of the rim (see Fig. 1-(a)). Accordingly, we establish $\left\{\boldsymbol{t}_{1}, \ldots, \boldsymbol{t}_{N_{R}}\right\}\left(\subset\left\{\boldsymbol{t}_{1}, \ldots, \boldsymbol{t}_{N_{E}}\right\}\right)$ as the subset of rooftop functions required for testing purposes over the rim mesh. We do not apply the field condition (2) as such over rectangular subdomains associated with mesh edges inside the top or bottom faces of the plate. Instead, we apply two electric-field conditions over the mid-surface $S_{\mathrm{M}}$, located inside the plate and equidistant from the top and bottom faces (see Fig. 1 -(a)); 


$$
\begin{aligned}
\left.\tilde{\boldsymbol{E}}^{s}(\boldsymbol{r})\right|_{\tan , \boldsymbol{r} \in S_{M}} & =-\left.\boldsymbol{E}^{i n c}(\boldsymbol{r})\right|_{\tan , \boldsymbol{r} \in S_{M}} \\
\frac{\partial}{\partial z}\left(\left.\tilde{\boldsymbol{E}}^{s}(\boldsymbol{r})\right|_{\tan , \boldsymbol{r} \in S_{M}}\right) & =-\frac{\partial}{\partial z}\left(\left.\boldsymbol{E}^{i n c}(\boldsymbol{r})\right|_{\tan , \boldsymbol{r} \in S_{M}}\right)
\end{aligned}
$$

where the variable $z$ evolves normally with respect to $S_{\mathrm{M}}(z=0)$.

Our thick-plate implementation relies on a rectangular tessellation over $S_{\mathrm{M}}$. This mesh is actually a translated copy of the top-face or bottom-face meshes, assumed identical. The field conditions in (3) and (4) are then tested with a set of rooftop basis functions $\left\{\boldsymbol{t}_{1}^{M} \ldots \boldsymbol{t}_{N_{M}}^{M}\right\}$ associated with the $N_{M}$ interior edges of the mesh at $S_{\mathrm{M}}$. In view of (2), (3) and (4), the resulting MoM-system yields

$$
\begin{gathered}
\iint_{Q_{r}} \boldsymbol{t}_{r} \cdot \tilde{\boldsymbol{E}}^{s}(\boldsymbol{r}) d S=-\iint_{Q_{r}} \boldsymbol{t}_{r} \cdot \boldsymbol{E}^{i n c}(\boldsymbol{r}) d S \\
\iint_{Q_{m}^{M}} \boldsymbol{t}_{m}^{M} \cdot \tilde{\boldsymbol{E}}^{s}(\boldsymbol{r}) d S=-\iint_{Q_{m}^{M}} \boldsymbol{t}_{m}^{M} \cdot \boldsymbol{E}^{i n c}(\boldsymbol{r}) d S \\
\iint_{Q_{m}^{M}} \boldsymbol{t}_{m}^{M} \cdot \frac{\partial}{\partial z}\left(\tilde{\boldsymbol{E}}^{s}(\boldsymbol{r})\right) d S=-\iint_{Q_{m}^{M}} \boldsymbol{t}_{m}^{M} \cdot \frac{\partial}{\partial z}\left(\boldsymbol{E}^{i n c}(\boldsymbol{r})\right) d S \\
1 \leq r \leq N_{R}, \quad 1 \leq m \leq N_{M}
\end{gathered}
$$

where $Q_{m}^{M}$ represents the duplet of rectangular facets that share the $m$-th edge inside the mesh at $S_{\mathrm{M}}$.

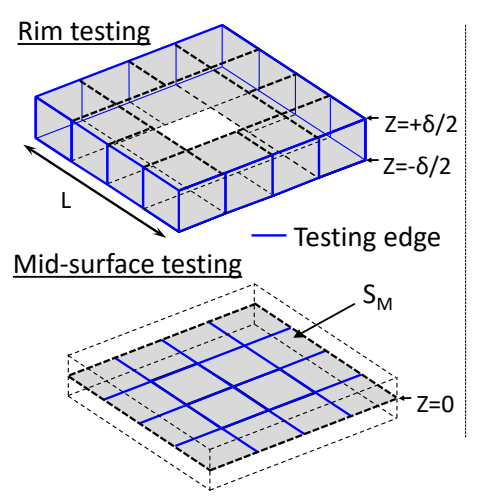

(a) $\underline{\text { z-symmetric expansion }}$

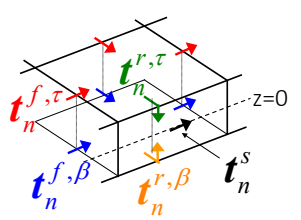

(b)
Figure 1. Thick-plate implementation for a full mesh of 96 edges $\left(N_{S}=16 ; N_{R B}=16 ; N_{R}=48 ; N_{M}=24\right)$.

Our meshes are defined symmetric with respect to $S_{\mathrm{M}}$. In the current expansion, hence, each rooftop at the upper half of the plate has a mirror rooftop at the lower half. We sort out the set of rooftop functions over the faces and over the rim, into two subsets of top $(\tau)$ and bottom $(\beta)$ functions, respectively, $\left\{\boldsymbol{t}_{1}^{f, \tau / \beta}, . ., \boldsymbol{t}_{N_{M}}^{f, \tau / \beta}\right\}$ and $\left\{\boldsymbol{t}_{1}^{r, \tau / \beta}, . ., \boldsymbol{t}_{N_{B}}^{r, \tau / \beta}\right\}$, so that each function is listed in the same order as its z-symmetric peer (see Fig-1-(b)). Inside the meshed rim a group of $N_{S}$ rooftops $\left\{\boldsymbol{t}_{n}^{s}\right\}$ is defined along the z-symmetry plane. These may be $\mathrm{z}$ - or xyoriented, depending on the mesh, and have no z-symmetric peer. The z-symmetric rooftops can be rearranged into two sets $\left\{\boldsymbol{b}_{n}^{\Sigma}\right\}$ and $\left\{\boldsymbol{b}_{n}^{\Delta}\right\}$, so that

$$
\begin{array}{cc}
\boldsymbol{b}_{n}^{\frac{\Sigma}{\Delta}}=\left(\boldsymbol{t}_{n}^{r, \tau} \pm \boldsymbol{t}_{n}^{r, \beta}\right) & 1 \leq n \leq N_{B} \\
\boldsymbol{b}_{n}^{\frac{\Sigma}{\Delta}}=\left(\boldsymbol{t}_{n-N_{B}}^{f, \tau} \pm \boldsymbol{t}_{n-N_{B}}^{f, \beta}\right) & N_{B}<n \leq N_{B}+N_{M}
\end{array}
$$

For a mesh with an xy-oriented subset $\left\{\boldsymbol{t}_{n}^{s}\right\}$, as in Fig. 1, the disjoint union of this set with $\left\{\boldsymbol{b}_{n}^{\Sigma}\right\}$ in the scattered-field expansion in (1) together with the field boundary condition (6), the field condition derived from adding the outcomes of (5) for the top- and bottom-rim subsets and the condition (5) particularized for $\left\{\boldsymbol{t}_{n}^{s}\right\}$ leads to a system with a unique solution. The set $\left\{\boldsymbol{b}_{n}^{\Delta}\right\}$ along with the field condition (7) and the outcome of subtracting (5) when particularized for the topand bottom-rim subsets also provides a uniquely solvable system. Therefore, an original problem with dimension $\mathrm{Ne}=$ $2 N_{M}+2 N_{B}+N_{S}$ is converted into two uncoupled matrix systems with dimensions $N_{M}+N_{B}+N_{S}$, if the former, and $N_{M}+N_{B}$, if the latter. This is computationally more efficient, especially for a big amount of unknowns, and can be easily parallelized.

\section{RESULTS AND DISCUSSION}

In Fig. 2, we show the computed RCS for a $1 \lambda \times 1 \lambda$ plate with $0.1 \lambda$ thickness. Whereas the thin-plate approach turns out very inaccurate, the full MoM-solution and our thick-plate formulation exhibit very similar accuracy.

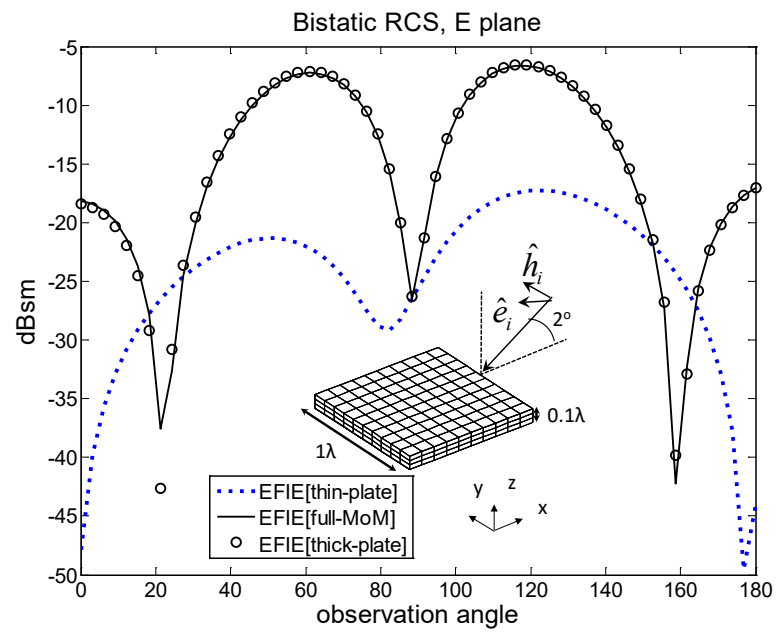

Figure 2. Computed RCS for a $1 \lambda \times 1 \lambda$ PEC-plate under an oblique transversal-magnetic incidence $(\lambda=1 \mathrm{~m}) . \quad N_{E}=640$; $N_{S}=40 ; N_{B}+N_{M}=300$

\section{ACKNOWLEDGEMENT}

This work was supported by FEDER and the Spanish Comisión Interministerial de Ciencia y Tecnología (CICYT) 
under projects TEC2016-78028-C3-1-P, TEC2017-84817-C22-R and the Unidad de Excelencia Maria de Maeztu MDM2016-0600.

\section{REFERENCES}

[1] Branko M. Kolundzija and Antonije R. Djordjevic, Electromagnetic Modeling of Composite Metallic and Dielectric Structures, Boston: Artech House, 2002.
[2] Edward H. Newman and Mark R. Schrote, "On the Current Distribution for Open Surfaces," IEEE Trans. Antennas Propag., vol. AP-31, no. 3, pp. 515-518, May 1983

[3] Allen W. Glisson, and Donald R. Wilton, "Simple and Efficient Numerical Methods for Problems of Electromagnetic Radiation and Scattering from Surfaces", IEEE Trans. Antennas Propag., vol. AP-28, no. 5, pp. 593-603, Sept. 1980 